\title{
El desarrollo típico y atípico de la subordinación en español. Estudio en dos poblaciones*
}

\author{
The typical and atypical development of subordination in \\ spanish. Study of two populations
}

Nina Crespo ${ }^{* *}$, Carola Alvarado ${ }^{* * *}$, Jeannette Sepúlveda ${ }^{* * * *}$, Alejandra Figueroa-Leighton ${ }^{* * * *}$

\begin{abstract}
RESUMEN
Los niños con trastorno específico del lenguaje evidencian una alteración en el procesamiento de la subordinación. Interesa examinar aquí el aumento de dichas estructuras y el registro de los fallos que producen estos niños a medida que crecen respecto de sus pares con desarrollo típico. Para ello, se planteó un estudio longitudinal, descriptivo-comparativo con 50 estudiantes (25 TEL, 25 DT) quienes recontaron tres cuentos. El corpus fue recolectado en tres momentos diferentes de su escolarización, distribuidos en dos años (cinco, seis y siete años). En el análisis se aplicaron dos índices: productividad de subordinación y desviaciones interclausulares en la subordinación y se identificaron diferentes desviaciones interclausulares: sustitución, incorporación, elisión y discordancia. Los resultados permiten observar que el grupo TEL aumenta el uso de subordinación y las desviaciones interclausulares respecto de sus pares con desarrollo típico.
\end{abstract} Palabras clave: trastorno específico del lenguaje, desarrollo típico, subordinación, estudio longitudinal, comparativo y descriptivo.

\footnotetext{
Estudio inserto en el marco del Proyecto Fondecyt 1160653.

** Chilena. Doctora en Lingüística. Pontificia Universidad Católica de Valparaíso, Valparaíso, Chile.nina.crespo@pucv.cl

*** Chilena. Doctora@ en Lingüística. Pontificia Universidad Católica de Valparaíso, Valparaíso, Chile. carola.alvarado@pucv.cl

***** Chilena. Magíster en Lingüística Aplicada. Pontificia Universidad Católica de Valparaíso, Valparaíso, Chile. jeannette.sepulveda@pucv.cl

Chilena. Fonoaudióloga, Doctora@ en Lingüística. Universidad de Valparaíso, Valparaíso, Chile. alejandra.figueroa@uv.cl
} 


\begin{abstract}
Children with specific language impairment (SLI) show an alteration in the processing of subordination. It is interesting to examine here the increase in these structures and the number of failures produced by these children as they grow up, with respect to their peers with TD. To do observe this, a longitudinal, descriptive-comparative study was applied to 50 students -25 SLI, 25 TD - who were asked to tell three stories. The corpus was collected at three different times during their schooling distributed over two years (five, six and seven years old). In the analysis, two indices were applied: that of subordination productivity and that of interclausal deviations in subordination. Moreover, different types of interclausal deviations were identified, including substitution, incorporation, elision, and disagreement. The results have allowed us to observe that, ontogenetically, the SLI group increases the use of subordination and interclausal deviations with respect to their TD peers.
\end{abstract} Keywords: specific language impairment, children with typical development, subordination, longitudinal, descriptivecomparative study. 


\section{Introducción}

En los estudios que se han llevado a cabo en niños diagnosticados con trastorno específico del lenguaje (TEL) -comparándolos con niños de desarrollo típico (DT) - se ha observado que los niños con TEL presentan dificultades para construir y comprender una sintaxis compleja, específicamente referida al uso de mecanismos de vinculación clausular como lo es la subordinación (Van der Lely, 1998, 2005). Por una parte, se ha sostenido que cuando producen textos - orales o escritos- los menores con TEL construyen menos oraciones subordinadas que sus pares con DT (Coloma, 2013), así como también que, al construir estructuras subordinadas, producen cuantitativa y cualitativamente más errores de tipo formal que los niños con DT (Van der Lely, 1998, 2005).

Diversos trabajos han permitido lograr un esbozo del desempeño gramatical en la subordinación de los niños con TEL hablantes de español (Acosta, Moreno y Axpe, 2014; Coloma, Araya, Quezada, Pavez y Maggiolo, 2016; Coloma, Mendoza y Carballo, 2017; Jackson-Maldonado y Maldonado, 2015). Sin embargo, aún no se han esclarecido ciertos aspectos del déficit. Por ejemplo, por un lado los hallazgos obtenidos por los diferentes estudios no corresponden a datos longitudinales a partir de un seguimiento de un mismo grupo de sujetos, sino solo evidencian los resultados de la comparación de grupos diferentes de distintas edades; y, por otra parte, si bien hay estudios en los cuales se describen los problemas gramaticales de los niños TEL (Acosta et al., 2014; Auza y Morgan, 2013; Jackson-Maldonado \& Maldonado, 2017), no se han focalizado de forma particular los fallos gramaticales que tienen lugar en la vinculación clausular de oraciones subordinadas. Por consiguiente, en este estudio se describirá el desarrollo longitudinal de la subordinación en una única población TEL, comparándola con la que evidencia una única población con DT que posee características demográficas similares. Para concretar la investigación nos propusimos tres principales objetivos:

- Determinar si evolutivamente la productividad de la subordinación se mantiene cuantitativamente deficitaria en los niños con TEL en comparación con sus pares con DT.

- Establecer si existe diferencia en el porcentaje de vínculos interclausulares con fallos o desviaciones en las narraciones producidas 
por niños con TEL y DT medidos en tres momentos de su escolarización.

- Determinar el tipo de error que tiene lugar en la subordinación en las narraciones de los niños con TEL comparado con las narraciones de la población con DT.

\section{La subordinación en el español}

La subordinación es un fenómeno concreto que nos permite reconocer y analizar aspectos fundamentales del lenguaje. Tradicionalmente, el término se ha aplicado para describir la relación que se establece entre un elemento subordinado y uno principal o subordinante. Bajo estas premisas teóricas, la oración subordinada establece una relación jerárquica entre una cláusula subordinada y otra superordinada —la principal- la que, a su vez, puede estar subordinada a un nivel más alto en la jerarquía de inclusión clausular (Mota, 2015).

Según la Nueva gramática de la lengua española (Real Academia Española/Asociación de Academias de la Lengua Española, RAE/ASALE, 2009), las cláusulas subordinadas en español pueden ser clasificadas de acuerdo con la función sintáctica que cumplen en la oración principal, o bien, con el criterio estructural, es decir, los rasgos de su verbo. Así, la subordinada puede funcionar como un sintagma nominal (SN), adjetival (SAdj) o adverbial (SAdv), como se ve en (1).

\begin{tabular}{lll}
\hline (1) & a. Me alegra [que hayas llegado a casa] & [subordinada sustantiva] \\
\hline $\begin{array}{l}\text { b. Necesito una secretaria [que sea } \\
\text { bilingüe] }\end{array}$ & [subordinada adjetiva] \\
\hline c. Lo llamaré [cuando llegue a casa] & [subordinada adverbial] \\
\hline
\end{tabular}

Respecto del punto de vista estructural, las subordinadas pueden construirse con un verbo: (a) finito con terminación desinencial que indica el sujeto, o (b) no finito con un verboide (infinitivo, participio o gerundio). Esto se grafica en las alternancias de (2), donde el cambio de las formas (finita/no finita) produce cambio en la asignación del sujeto respecto del cual está predicando el verbo.

\begin{tabular}{lll}
\hline (2) a. Juan compró zapatillas nuevas para caminar & [Forma No Finita] \\
cómodo (Juan) & \\
\hline $\begin{array}{l}\text { b. Juan compró zapatillas para que camine } \\
\text { cómodo (el hijo de Juan) }\end{array}$ & [Forma Finita] \\
\hline
\end{tabular}


Ahora bien, a partir de estas clasificaciones tanto funcionales como estructurales, es posible establecer una serie de restricciones morfosintácticas que posibilitan este tipo de relación gramatical. Estas propiedades son: desde el punto de vista estructural, presencia o ausencia de nexo y características TAM (tiempo, aspecto y modo); y, desde el punto de vista funcional, presencia o ausencia de preposición junto al nexo. Así, en (3) se puede observar cómo las formas finitas o no finitas determinan la presencia o ausencia del nexo, mientras que en (4) se muestra cómo la presencia o ausencia de preposición indica que la subordinada está cumpliendo una determinada función sintáctica.

(3) a. No le gusta que lo molesten por [con nexo + Forma Finita] tonteras.

b. No le gusta ser molestado por [sin nexo + Forma No Finita] tonteras.

c. "Dice que subir la caja.

[con nexo + Forma Finita]

(4) a. Me alegro de que hayas venido. [subordinación sustantiva, término preposicional]

b. Me alegra que hayas venido. [subordinación sustantiva, sujeto]

Otro aspecto importante desde el punto de vista estructural refiere al vínculo o determinación entre las formas verbales que están involucradas en la relación clausular de subordinación - características TAM-. En este vínculo existe una determinación del verbo principal respecto del verbo que nuclea a la cláusula subordinada. Así, mientras la oración (6.a) es gramatical, no ocurre lo mismo con (6.b) y (6.c), ya que en el primer caso se usa el modo indicativo en vez de la forma subjuntiva requerida por el verbo "querer"; $y$, en el segundo caso, si bien se usa subjuntivo en la subordinada, existe una discordancia en el tiempo del verbo respecto del verbo de la principal.

(6) a. Mamá no quiere que salgas.

b. "Mamá no quiere que sales.

c. "Mamá no quiere que salieras.

Según Perea y Martínez-Atienza (2013), este fenómeno denominado la consecutio temporum es un tipo especial de relación anafórica entre los tiempos verbales y corresponde a una regla por la cual el verbo de la cláusula subordinada está condicionado por el verbo 
de la cláusula principal y no por el momento de la enunciación. Este condicionamiento afecta sobre todo a la subordinación sustantiva (Carrasco, 1999) y adverbial (Veiga y Mosteiro, 2006) y está delimitado por diversos factores entre los que se incluyen el sistema de tiempos verbales (Rojo y Veiga, 1999) y las restricciones léxicas del verbo principal.

\section{Trastorno específico del lenguaje y subordinación}

El TEL se define como una alteración significativa en la adquisición y desarrollo del lenguaje, que no se justifica por causa física, neurológica, intelectual ni sensorial, en condiciones sociales adecuadas (Aguado, Coloma, Martínez, Mendoza y Montes, 2015). En Chile, un $4 \%$ de los niños entre tres y siete años de edad evidencia el trastorno y se presenta con mayor frecuencia en varones (Villanueva, De Barbieri, Palomino y Palomino, 2008).

La población con TEL tiene diferentes perfiles de déficits de lenguaje y gravedad; esta variabilidad ha sido referida por diversos autores (Aguado, 1999; Leonard, 2014a; Mendoza, 2001; Van der Lely, 1998, 2005). Respecto de sus habilidades lingüísticas, los niños pueden presentar un rango amplio de alteraciones en el procesamiento del lenguaje, dependiendo del nivel lingüístico (fonético, fonológico, morfológico, sintáctico, semántico o pragmático), o bien, de la habilidad de lenguaje utilizada (comprensión y/o producción) que puede estar selectivamente afectada (Leonard, 2014a), lo cual evidencia que no se trata de una condición homogénea. No obstante, existe consenso en que determinadas dimensiones del lenguaje muestran especial déficit y podrían considerarse como marcadores del trastorno. Tal sería el caso de ciertos aspectos gramaticales como la complejidad sintáctica (Leonard, 2014a; Van der Lely, 2005), tema que ha sido objeto de varias investigaciones que han estudiado la producción de oraciones complejas en niños con TEL en diferentes lenguas.

En concreto, un estudio acerca de la subordinación en niños hablantes de alemán concluyó que, en el caso de la producción de oraciones subordinadas relativas, los menores con TEL de entre cuatro y 10 años producían una cantidad mucho menor de este tipo de oraciones que sus pares en edad con DT y que, aunque se estimuló la producción de este tipo de oraciones, los niños con TEL las evita- 
ban, prefiriendo estructuras simples. En cuanto a la calidad de dichas construcciones, se observó que casi la mitad de la cantidad producida era gramatical y contextualmente correcta, y que estas cualidades se asentaban con la edad (Adani, Stegenwallner-Schütz, Haendler \& Zukowski, 2016). En el caso de la lengua inglesa, un estudio realizado en niños de entre siete y nueve años y que tenía el propósito de estudiar la relación entre contenido y forma en las narrativas de niños con TEL, observó que estos producían historias con contenido pobre, pero gramaticalmente correctas, o bien, producían historias más elaboradas, pero con descuidos en la gramática (Colozzo, Gillam, Wood, Schnell \& Johnston, 2011).

En otro estudio, cuyo propósito era analizar la gramaticalidad y complejidad de oraciones en niños hablantes de español de seis años, se observó que los niños con TEL presentaban más errores gramaticales que los niños con DT de igual edad o menores. En relación con la complejidad de las oraciones no se encontraron diferencias significativas entre los grupos (Coloma et al., 2016). No obstante, en el trabajo realizado por Alfaro-Faccio, Crespo y Alvarado (2016) sí se evidenciaron diferencias en la complejización del discurso narrativo oral entre niños hispanohablantes con TEL y DT. En efecto, esta investigación demostró que los menores con TEL presentaban una arquitectura interclausular diferente, privilegiando el uso de oraciones coordinadas por sobre las subordinadas y, con ello, un discurso más simple y menos informativo. Los estudios mencionados evidencian, en su mayoría, que en distintas lenguas y edades la subordinación representa una dificultad en el procesamiento sintáctico de los niños con TEL si se los compara con sus pares normotípicos.

Sin embargo, a nuestro entender, estos estudios presentan una limitación. Los investigadores se concentraron en la comparación del desempeño de niños con TEL y niños con DT, sin observar los posibles cambios que pueden producirse a medida que estos niños crecen y se adentran en la experiencia escolar - $\mathrm{y}$, en el caso de los niños con TEL, experimentan también el apoyo terapéutico-. Dar cuenta de estos cambios no es un tema menor, ya que en opinión de Nippold (2004) al estudiar el desarrollo tardío del lenguaje (después de los cinco años) destaca el incremento de formas y el mayor uso de subordinación como una pieza clave en la sintaxis de niños con DT. Cabe preguntarse, 
entonces, cómo se produce este fenómeno en niños con TEL y si existen diferencias en la forma en que se desenvuelven las poblaciones.

Otro aspecto interesante de considerar está relacionado con la manera en la que se observará el fenómeno de la subordinación. En este sentido, los estudios citados comparan el uso de estructuras y observan no solo las cantidades, sino los diferentes tipos que se utilizan (Acosta et al., 2014; Buiza, Adrián, González y Rodríguez-Parra, 2004; Hincapié et al., 2008). Sin embargo, aquí hemos decidido además considerar otro aspecto de la subordinación: las desviaciones en la construcción. Se entiende por desviación aquellos fallos o usos poco frecuentes, como ciertas formas de hipérbaton, que son sistemáticos (ocurren en los mismos espacios estructurales), pero no consistentes (esto es, ocurren algunas veces y otras no en el mismo discurso) que cometen los niños, con TEL o con DT, cuando construyen una determinada estructura sintáctica y que son significativamente más abundantes en los niños con TEL.

En otros trabajos (Crespo, Alfaro-Faccio, Góngora, Alvarado y Marfull, 2020), se distinguen seis categorías de desviaciones en diversas estructuras sintácticas:

- Discordancia: no congruencia entre los morfemas de género, número, tiempo, aspecto y modo.

- Elisión: omisión de un elemento léxico o funcional que es exigido por la estructura sintáctica.

- Sustitución: reemplazo de un elemento funcional o léxico exigido por una estructura sintáctica, por otro elemento con el cual comparte algunos rasgos, sin ser este último contextualmente adecuado.

- Incorporación: adición de un elemento léxico o funcional que no es exigido por la estructura sintáctica y no es contextualmente adecuado.

- Hipérbaton: cambio del orden de las palabras y/o sintagmas que es predominante en una lengua configuracional.

- Anomalías morfológicas, hiperregularización verbal.

Este trabajo se propuso observar cuáles de estas desviaciones operaban en el ámbito de la subordinación, especialmente en la conexión entre la oración principal y la subordinada. 


\section{Metodología}

La presente investigación se enmarca dentro de un estudio longitudinal $^{1}$ de alcance descriptivo-comparativo que se aplicó en dos poblaciones de niños con TEL y con DT para observar el desarrollo de su productividad sintáctica y la presencia de desviaciones a nivel gramatical.

\subsection{Participantes}

Se reclutaron 50 estudiantes de colegios con sistema regular, con diagnóstico de TEL ( $\mathrm{N}=25$; 16 hombres y 9 mujeres) y con desarrollo típico $(\mathrm{N}=25 ; 13$ hombres y 12 mujeres), los que fueron evaluados en diversas oportunidades.

Para este estudio tomamos solo el tiempo 1 (esto es, entre los cinco años y cinco años 11 meses), el tiempo 3 (entre los seis años y seis años 11 meses) y el tiempo 5 (entre siete años y siete años 11 meses de edad).

Los niños del grupo DT tenían un desempeño cognitivo y lingüístico adecuado a su edad y su escolarización seguía los estándares normales, según el criterio de la educadora a cargo del nivel y del gabinete psicopedagógico de cada establecimiento educacional. La selección de los niños del grupo TEL se efectuó de acuerdo con las normas chilenas para su diagnóstico (Decreto $\mathrm{n}^{\circ} 170$ ), y fue realizado mediante el test exploratorio de gramática española de Toronto (Pavez, 2010), tanto la subprueba receptiva (STSG-R) como la expresiva (STSG-E) y el TEPROSIF-R (Pavez, Maggiolo y Coloma, 2009). En ambas pruebas, los sujetos que obtuvieron 1,5 de desviación estándar bajo la norma fueron considerados con rendimiento deficitario e ingresaron al grupo TEL. Además, para corroborar que presentaban un desempeño cognitivo y auditivo dentro de la norma se aplicó el test de matrices progresivas-escala coloreada (Raven, 2005), tomando la norma chilena (Ivanovic et al., 2000), más una audiometría tonal e impedanciometría. En la Tabla 1 se presenta una síntesis de las mediciones realizadas y sus resultados.

El estudio fue validado por el Comité de Bioética de la Pontificia Universidad Católica de Valparaíso, Chile, con fecha 8 de mayo de 2013. 
Tabla 1.

Resultados de las evaluaciones diagnósticas y de selección del grupo TEL.

\begin{tabular}{lcc}
\hline & Media & $\begin{array}{c}\text { Desviación } \\
\text { Estándar }\end{array}$ \\
\hline Pruebas & & \\
\hline STSG-E & 23,61 & 6,45 \\
\hline STSG-R & 22,78 & 7,40 \\
\hline TEPROSIF-R & 28,94 & 15,87 \\
\hline Test de matrices coloreadas de Raven & 20,89 & 3,77 \\
\hline Evaluación auditiva & Min. & Max. \\
\hline $\begin{array}{l}\text { Audiometría tonal. Promedio tonal puro oído } \\
\text { derecho }\end{array}$ & 8,3 & 23,3 \\
\hline $\begin{array}{l}\text { Audiometría tonal. Promedio tonal puro oído } \\
\text { izquierdo }\end{array}$ & 8,3 & 21,7 \\
\hline
\end{tabular}

Fuente: Elaboración propia.

\subsection{Instrumentos y recolección}

Para la elicitación de los 150 textos que son parte del corpus NIR (2014), se usó una tarea de recontado. Para ello, se utilizaron tres textos narrativos audiovisuales que fueron aplicados en diferentes momentos (cinco, seis y siete años de edad de los sujetos). El primero fue La mariposa Flopi; el segundo, Bato, el oso futbolista; y, el tercero, El valiente Lilo; los que fueron elaborados por Crespo, Góngora y Manghi (2013) y se construyeron siguiendo la gramática de las historias de Stein y Glenn (1979), Peterson y McCabe (1983), Owens (1995) y Pavez, Coloma y Maggiolo (2008). Los tres relatos mantuvieron la estructura de establecimiento de escena, dos episodios y reacción-resolución. Formalmente, estaban compuestos por 650 palabras y 40 cláusulas en promedio.

La dinámica de dos interlocutores en la aplicación del instrumento y recolección de los datos tuvo como finalidad recrear una situación de relato infantil pragmáticamente verosímil y cercana a su realidad ${ }^{2}$. 


\subsection{Análisis}

Los 150 discursos narrativos orales fueron transcritos fonéticamente. La información lingüística fue analizada sintácticamente en: separación de cláusulas; identificación de cláusulas subordinadas; e identificación de desviaciones en las vinculaciones interclausulares. Estas unidades fueron contabilizadas y, además, se calcularon índices y proporciones.

\subsubsection{Unidades de análisis}

Las primeras dos unidades de análisis consideradas fueron la cláusula y la cláusula subordinada. Se entenderá por cláusula a la unidad sintáctica que está regida por un verbo núcleo (finito o no finito) que contiene un predicado expresando una única acción o estado (Berman \& Slobin, 1994). Respecto de la cláusula subordinada, se asume como una unidad regida por relaciones de dependencia e incrustación clausular. Para el español, la Nueva gramática de la lengua española (RAE/ ASALE, 2009) ha establecido ciertos tipos de subordinación identificadas, principalmente, a partir de la función sintáctica que cumple la cláusula dependiente e incrustada — subordinada - respecto de la principal o subordinante.

La tercera unidad de análisis corresponde a la desviación en la subordinación (DS). Como ya se señaló, se entiende por desviación a aquellos fallos o usos poco frecuentes, como ciertas formas de hipérbaton, que son sistemáticos (ocurren en los mismos espacios estructurales), pero no consistentes (ocurren algunas veces y otras no en el mismo discurso). La desviación interclausular corresponderá, por tanto, a aquel fallo ocasional en el cual no se cumplen las reglas formales propias de las relaciones interclausulares que rigen la subordinación en español, por ejemplo, la concordancia verbal entre cláusulas - ya sea, tiempo o modo-, elisión o sustitución del subordinante. A partir de las seis categorías de desviaciones planteadas en el marco teórico, se consideraron cuatro tipos de desviaciones que afectan el vínculo interclausular en la subordinación. Estos son: sustitución (verboide, nexo, formas verbales y preposición); incorporación (de nexo y preposición); elisión (de nexo, preposición, subordinada y verbo) y discordancia (de tiempo, modo y aspecto del verbo, y la persona del pronombre). 
En la Tabla 2 se detallan ejemplos concretos, tomados del corpus, de cada una de estas desviaciones.

Tabla 2.

Codificación de la categoría desviación.

\begin{tabular}{|c|c|c|}
\hline \multicolumn{2}{|c|}{$\begin{array}{l}\text { Tipo de desviación } \\
\text { interclausular }\end{array}$} & \multirow{2}{*}{$\begin{array}{l}\text { Ejemplo } \\
\begin{array}{l}\text { se tiró sin gritar/el otro con gritar } \\
\text { (BATO_06TEL) }\end{array}\end{array}$} \\
\hline Sustitución & Verboide & \\
\hline & Nexo & pensó si se podría caer (LILO_44TEL) \\
\hline & $\begin{array}{l}\text { Formas } \\
\text { verbales }\end{array}$ & $\begin{array}{l}\text { y después se sintió/ triste llorando } \\
\text { (BATO_15DT) }\end{array}$ \\
\hline & Preposición & $\begin{array}{l}\text { pero un día Lilo tenía mucho miedo en } \\
\text { caerse (LILO_15DT) }\end{array}$ \\
\hline Incorporación & $\begin{array}{l}\text { Nexo y/o } \\
\text { preposición }\end{array}$ & $\begin{array}{l}\text { pero dijo no voy a tener de miedo/ } \\
\text { (LILO_06TEL) }\end{array}$ \\
\hline \multirow[t]{4}{*}{ Elisión } & Nexo & $\begin{array}{l}\text { y primero sus/sus amigos fueron rescatarla } \\
\text { (FLOPI_07TEL) }\end{array}$ \\
\hline & Preposición & $\begin{array}{l}\text { y la serpiente le tuvo miedo escénico que le } \\
\text { pegue/(LILO_10TEL) }\end{array}$ \\
\hline & Subordinada & $\begin{array}{l}\text { y fue a su casa corriendo a buscar un /e/ } \\
\text { (FLOPI_DT67) }\end{array}$ \\
\hline & Verbo & $\begin{array}{l}\text { una vez un oso futbolista vivía en el bosque } \\
\text { cuando su amigo (BATO_14TEL) }\end{array}$ \\
\hline \multirow[t]{3}{*}{ Discordancia } & TAM & $\begin{array}{l}\text { conejo le dijo al oso que se vayaba de la } \\
\text { cancha (BATO_14TEL) }\end{array}$ \\
\hline & Persona & $\begin{array}{l}\text { pero un día le mostró cómo volaban } \\
\text { (LILO_52DT) }\end{array}$ \\
\hline & Pronominal & $\begin{array}{l}\text { y tuvo que hacer las zapatillas/ tanto } \\
\text { trabajo/ hasta que los terminó (BATO } \\
\text { 37TEL) }\end{array}$ \\
\hline
\end{tabular}

Fuente: Elaboración propia

\subsection{2. Índices}

El índice de productividad de subordinación (IPS) se ha construido a partir de la cantidad total de cláusulas subordinadas (TCS) y la cantidad de cláusulas totales (TC) producidas por cada menor en su tarea de recontado. El IPS se calcula dividiendo el TCS por el TC.

$$
\text { IPS }=(\text { TCS } / \text { TC })
$$


El índice de desviaciones interclausulares en la subordinación (IDS) permite cuantificar la proporción de fallos (TDS) respecto de la cantidad de vínculos interclausulares que implican subordinación (TCS), producidos por cada menor en su tarea de recontado. Este índice se calcula dividiendo el total de desviaciones identificadas por el total de cláusulas subordinadas.

$$
\text { IDS }=(\text { TDS } / T C S)
$$

\section{Resultados}

\subsection{Análisis estadístico de los resultados}

\subsubsection{Productividad de subordinación (PS)}

El análisis descriptivo en la Tabla 3 ilustra que durante la trayectoria temporal, el grupo DT evidenció mayor PS en una etapa inicial (M: 0,247) respecto del grupo TEL (M: 0,198). Esta disparidad se mantiene en un segundo momento, pues en el cuento de Bato se observa que el promedio de DT (M: 0,332) es mayor que el del grupo TEL (M: 0,270). No obstante, la discrepancia en el índice de productividad evidenciado en los dos primeros tiempos se equiparó en el último cuento - Lilo-, observándose que ambos grupos presentan el mismo IPS (M: 0,378) sin grandes diferencias en su desviación estándar.

Tabla 3.

Descriptivos del índice de productividad de subordinación de los grupos DT y TEL en la variable intrasujeto (medidas repetidas).

\begin{tabular}{lcccc}
\hline \multirow{2}{*}{ Medidas } & Grupo & Media & $\begin{array}{c}\text { Desviación } \\
\text { Estándar }\end{array}$ & $\mathrm{N}$ \\
\hline (Tiempo 1) IPS_Flopi & DT & 0,247 & 0,111 & 25 \\
\cline { 2 - 5 } & TEL & 0,198 & 0,105 & 25 \\
\hline (Tiempo 3) IPS_Bato & DT & 0,332 & 0,087 & 25 \\
\cline { 2 - 5 } & TEL & 0,270 & 0,096 & 25 \\
\hline (Tiempo 5) IPS_Lilo & DT & 0,378 & 0,067 & 25 \\
\cline { 2 - 5 } & TEL & 0,378 & 0,077 & 25 \\
\hline
\end{tabular}

Fuente: Elaboración propia. 
Al comparar la trayectoria de ambos grupos (Tabla 4) no se evidencia un efecto de interacción entre los factores inter e intrasujeto $(F(2,96)=1,693, p=0,189)$. Es decir, no se observa una diferencia estadísticamente significativa en la progresión temporal entre ambos grupos.

Tabla 4.

Análisis de interacción entre las variables medidas y grupo en la productividad de subordinación.

\begin{tabular}{lcccccc}
\hline & $\begin{array}{c}\text { Suma de } \\
\text { cuadrados }\end{array}$ & df & $\begin{array}{c}\text { Media } \\
\text { cuadrática }\end{array}$ & $F$ & $P$ & $\eta^{2}{ }_{p}$ \\
\hline Medidas & 0.607 & 2 & 0,303 & 38,336 & $<, 001$ & 0,444 \\
\hline $\begin{array}{l}\text { Medidas } * \\
\text { grupo }\end{array}$ & 0.027 & 2 & 0,013 & 1,693 &, 189 & 0,034 \\
\hline Residual & 0.760 & 96 & 0,008 & & & \\
\hline
\end{tabular}

Nota: df significa grados de libertad, del inglés degrees of freedom.

Fuente: Elaboración propia.

Los análisis permiten observar que, si bien los sujetos con TEL y con DT muestran avances significativos en la PS entre los distintos momentos de la toma, la trayectoria de ambos grupos es similar (Figura 1).

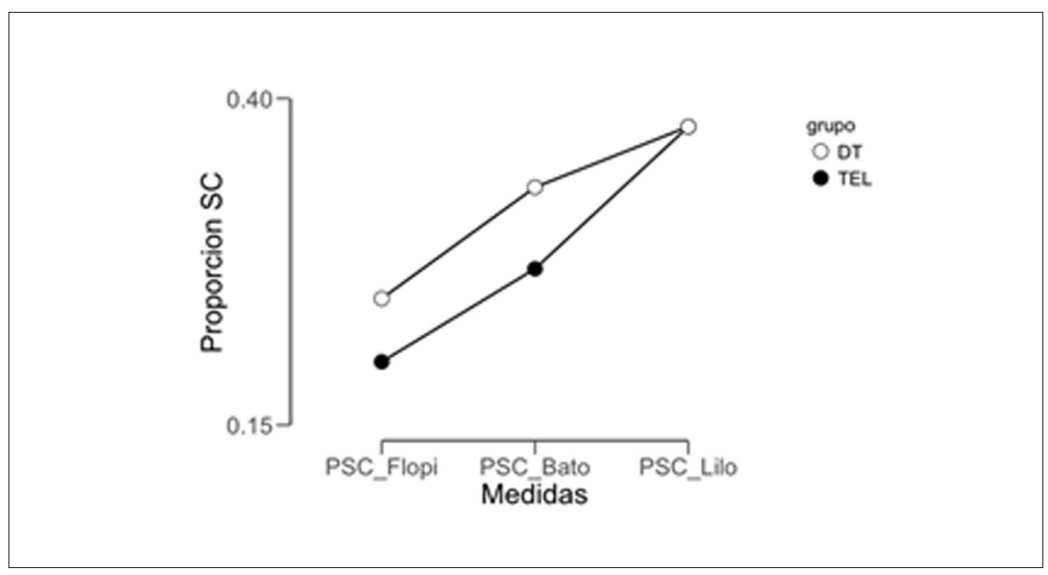

Figura 1. Progresión temporal del desempeño de los grupos DT versus TEL en el índice de productividad de subordinación (IPS).

Fuente: Elaboración propia. 


\subsubsection{Proporción de desviaciones en la subordinación}

En la Tabla 5 se presentan los estadísticos descriptivos que permiten comparar ambos grupos en el índice de desviaciones.

Tabla 5.

Descriptivos del índice de desviaciones en la subordinación de los grupos DT y TEL en la variable intrasujeto (medidas repetidas).

\begin{tabular}{lcccc}
\hline \multirow{2}{*}{ Medidas } & Grupo & Media & $\begin{array}{c}\text { Desviación } \\
\text { Estándar }\end{array}$ & N \\
\hline IDS_Flopi & DT & 0,092 & 0,115 & 25 \\
\cline { 2 - 5 } & TEL & 0,084 & 0,172 & 25 \\
\hline IDS_Bato & DT & 0,180 & 0,183 & 25 \\
\cline { 2 - 5 } & TEL & 0,232 & 0,241 & 25 \\
\hline IDS_Lilo & DT & 0,076 & 0,060 & 25 \\
\cline { 2 - 5 } & TEL & 0,196 & 0,162 & 25 \\
\hline
\end{tabular}

Fuente: Elaboración propia.

El análisis descriptivo en la Tabla 5 permite observar que durante la trayectoria temporal, a los cinco años, el grupo DT presenta un índice mayor en las desviaciones interclausulares (M: 0,092) que sus pares en edad con TEL (M: 0,084). Esta disparidad se invierte a los seis años en Bato y aumenta la diferencia entre ambos grupos, siendo el grupo TEL el que presenta una mayor ocurrencia de fallos (M: 0,232) en comparación con DT (M: 0,180). En la última medición, correspondiente a Lilo, a los siete años, la diferencia se acrecienta entre las poblaciones, observándose que en TEL (M: 0,196) las desviaciones son mucho más frecuentes que en DT $(0,076)$. Esto nos permite deducir que, si bien en una etapa inicial de la trayectoria tanto TEL como DT evidencian una proporción de desviaciones similares, a medida que los sujetos avanzan en edad, la proporción de desviaciones parece disminuir en un grupo y aumentar en el otro. 
Tabla 6.

Análisis de interacción entre las variables medidas y grupo en el índice de desviaciones en la subordinación.

\begin{tabular}{lllllll}
\hline & $\begin{array}{c}\text { Corrección } \\
\text { de esfericidad }\end{array}$ & $\begin{array}{c}\text { Suma de } \\
\text { cuadrados }\end{array}$ & df & $\begin{array}{c}\text { Media } \\
\text { cuadrática }\end{array}$ & $F$ & $p$ \\
\hline Medidas & $\begin{array}{l}\text { Greenhouse- } \\
\text { Geisser }\end{array}$ & 0,352 & 1,635 & 0,215 & 6,623 & 0,004 \\
\hline $\begin{array}{l}\text { Medidas } \\
\text { * grupo }\end{array}$ & $\begin{array}{l}\text { Greenhouse- } \\
\text { Geisser }\end{array}$ & 0,103 & 1,635 & 0,063 & 1,929 & 0,160 \\
\hline Residual & $\begin{array}{l}\text { Greenhouse- } \\
\text { Geisser }\end{array}$ & 2,552 & 78,481 & 0,033 & & \\
\hline
\end{tabular}

Nota: df significa grados de libertad, del inglés degrees of freedom.

Fuente: Elaboración propia.

El análisis comparativo entre sujetos permite determinar la interacción de ambos grupos en su productividad de cláusulas subordinadas durante los tres momentos evaluados. Para tal caso, en la Tabla 6 se evidencia que existe una diferencia estadísticamente significativa en cada grupo respecto de su propia trayectoria temporal, es decir, entre la toma de Flopi, Bato y Lilo. No obstante, no se registró una diferencia estadísticamente significativa en la interacción entre los grupos durante la trayectoria temporal. Esto permite establecer que si bien existen diferencias en las proporciones, los recorridos de las dos poblaciones son similares y, por lo tanto, no pueden considerarse dos caminos totalmente distintos, tal como se evidencia en la Figura 2.

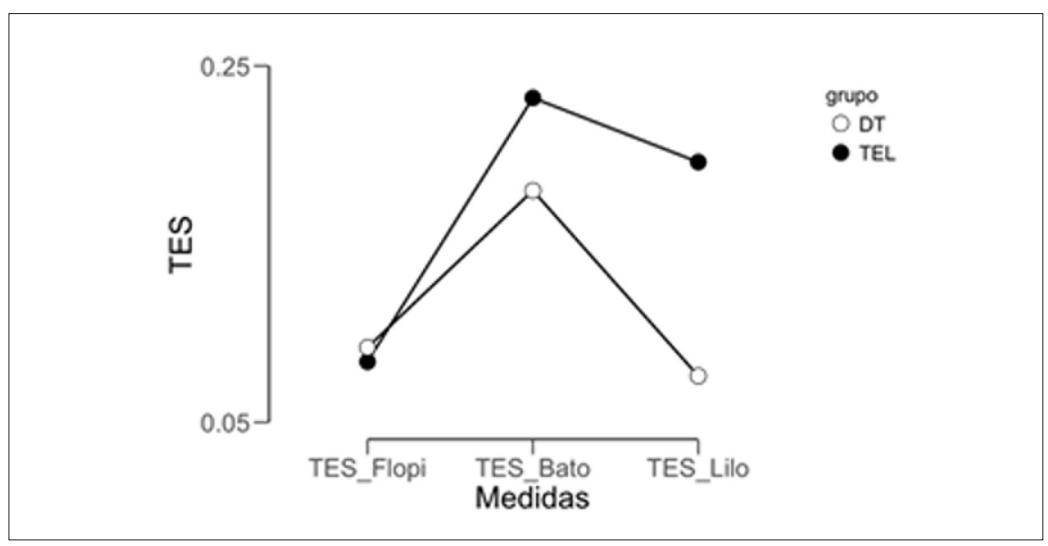

Figura 2. Progresión temporal del desempeño de los grupos DT versus TEL en la proporción de desviaciones en la subordinación (IDS). Fuente: Elaboración propia. 


\subsubsection{Total de desviaciones en la subordinación (TDS)}

En cuanto al total de desviaciones en la subordinación (TDS), en la Tabla 7 se presentan los estadísticos descriptivos que permiten comparar ambos grupos.

Tabla 7.

Descriptivos del total de desviaciones en la subordinación de los grupos DT y TEL en la variable intrasujeto (medidas repetidas).

\begin{tabular}{lcccc}
\hline \multicolumn{1}{c}{ Medidas } & Grupo & Media & Desviación Estándar & N \\
\hline \multirow{2}{*}{ TDS_Flopi } & DT & 0,520 & 0,653 & 25 \\
\cline { 2 - 5 } & TEL & 0,240 & 0,436 & 25 \\
\hline \multirow{2}{*}{ TDS_Bato } & DT & 1,160 & 1,106 & 25 \\
\cline { 2 - 5 } & TEL & 1,360 & 1,381 & 25 \\
\hline \multirow{2}{*}{ TDS_Lilo } & DT & 0,960 & 0,841 & 25 \\
& TEL & 2,480 & 1,960 & 25 \\
\hline
\end{tabular}

Fuente: Elaboración propia.

El análisis descriptivo permite observar que durante la progresión temporal, a los cinco años, el grupo DT presenta una mayor frecuencia de desviaciones (M: 0,520) que sus pares en edad con TEL (M: 0,240). Esta disparidad se invierte a los seis años en Bato y aumenta la diferencia entre ambos grupos, siendo el grupo TEL el que presenta una mayor ocurrencia de fallos (M: 1,360) en comparación con DT (M: 1,160). En la última medición, la diferencia se acrecienta entre las poblaciones, observándose que en TEL (M: 2,480) el total de desviaciones es mucho más frecuente que en DT $(0,960)$. Esto nos permite deducir que si bien en una etapa inicial de la trayectoria tanto TEL como DT evidencian una productividad similar en el total de desviaciones interclausulares, a medida que avanzan en edad ambas poblaciones se van diferenciando, siendo los niños TEL quienes aumentan considerablemente la cantidad de fallos.

El análisis comparativo entre sujetos ha permitido determinar la interacción de ambos grupos en el TDS durante los tres momentos evaluados. En la Tabla 8 se observa que la estimación del efecto de interacción resultó ser estadísticamente significativo $(F(2,96)=$ $17,579,8,236 ; p=0,001)$, es decir, ambos grupos hacen trayectorias distintas y, a la vez, las diferencias entre los tramos de edad son significativas para los dos grupos (Figura 3). Se evidencia que la 
magnitud de la diferencia de las trayectorias entre grupos es relevante $(\eta 2 p=0,146)$, de acuerdo con los criterios de Cohen (1988) para la interpretación de los tamaños del efecto.

Tabla 8.

Análisis de interacción entre las variables medidas y grupo en el total de desviaciones en la subordinación.

\begin{tabular}{cccccccc}
\hline & $\begin{array}{c}\text { Corrección } \\
\text { esfericidad }\end{array}$ & $\begin{array}{c}\text { Suma de } \\
\text { cuadrados }\end{array}$ & df & $\begin{array}{c}\text { Media } \\
\text { cuadrática }\end{array}$ & $F$ & $P$ & $\eta^{2}$ \\
\hline Medidas & $\begin{array}{c}\text { Greenhouse- } \\
\text { Geisser }\end{array}$ & $46,36^{\mathrm{a}}$ & $1,778^{\mathrm{a}}$ & $26,072^{\mathrm{a}}$ & $17,579^{\mathrm{a}}$ & $<, 001^{\mathrm{a}}$ & 0,268 \\
\hline $\begin{array}{c}\text { Medidas } \\
\text { * Grupo }\end{array}$ & $\begin{array}{c}\text { Greenhouse- } \\
\text { Geisser }\end{array}$ & $21,72^{\mathrm{a}}$ & $1,778^{\mathrm{a}}$ & $12,215^{\mathrm{a}}$ & $8,236^{\mathrm{a}}$ & $<, 001^{\mathrm{a}}$ & 0,146 \\
\hline Residual & $\begin{array}{c}\text { Greenhouse- } \\
\text { Geisser }\end{array}$ & 126,59 & 85,351 & 1,483 & & & \\
\hline
\end{tabular}

a Mauchly's test of sphericity indicates that the assumption of sphericity is violated $(p<.05)$. Nota: df significa grados de libertad, del inglés degrees of freedom.

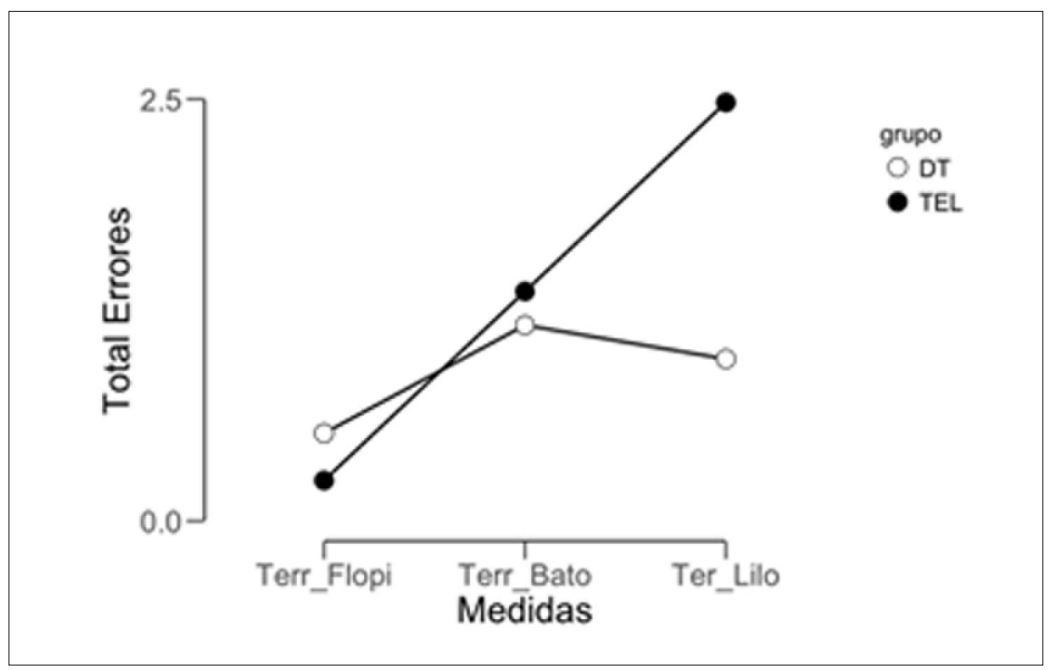

Figura 3. Progresión temporal del desempeño de los grupos DT versus TEL en total de desviaciones en la subordinación (TDS).

Fuente: Elaboración propia.

\subsubsection{Tipo de desviación en la subordinación}

Para ahondar en estos resultados, se describirá cómo se verifican estas trayectorias considerando los distintos tipos de desviación. 
a) Análisis descriptivo por tipo de desviación: sustitución, elisión, discordancia e incorporación

Respecto de la desviación sustitución (DS), elisión y discordancia, los resultados del análisis descriptivo muestran un comportamiento muy parecido al comparar las dos poblaciones (Tabla 9, 10 y 11 ).

Tabla 9.

Descriptivos de la desviación en la subordinación de tipo sustitución (DS) de los grupos DT y TEL en la variable intrasujeto (medidas repetidas).

\begin{tabular}{lcccc}
\hline \multirow{2}{*}{ Medidas } & Grupo & Media & $\begin{array}{c}\text { Desviación } \\
\text { Estándar }\end{array}$ & $\mathrm{N}$ \\
\hline (Tiempo 1) DS_Flopi & DT & 0,080 & 0,277 & 25 \\
\cline { 2 - 5 } & TEL & 0,040 & 0,200 & 25 \\
\hline (Tiempo 3) DS_Bato & DT & 0,480 & 0,653 & 25 \\
\cline { 2 - 5 } & TEL & 0,120 & 0,332 & 25 \\
\hline (Tiempo 5) DS_Lilo & DT & 0,320 & 0,627 & 25 \\
\cline { 2 - 5 } & TEL & 0,480 & 0,714 & 25 \\
\hline
\end{tabular}

Fuente: Elaboración propia.

Tabla 10.

Descriptivos de la desviación en la subordinación de tipo elisión (DE) de los grupos DT y TEL en la variable intrasujeto (medidas repetidas).

\begin{tabular}{lllll}
\hline \multirow{2}{*}{ Medidas } & \multicolumn{1}{c}{ Grupo } & Media & $\begin{array}{c}\text { Desviación } \\
\text { Estándar }\end{array}$ & N \\
\hline (Tiempo 1) DE_Flopi & DT & 0,200 & 0,408 & 25 \\
\cline { 2 - 5 } & TEL & 0,160 & 0,374 & 25 \\
\hline (Tiempo 3) DE_Bato & DT & 0,080 & 0,277 & 25 \\
\cline { 2 - 5 } & TEL & 0,280 & 0,458 & 25 \\
\hline (Tiempo 5) DE_Lilo & DT & 0,160 & 0,374 & 25 \\
\cline { 2 - 5 } & TEL & 0,800 & 1,155 & 25 \\
\hline
\end{tabular}

Fuente: Elaboración propia. 
Tabla 11.

Descriptivos de la desviación en la subordinación de tipo discordancia (DD) de los grupos DT y TEL en la variable intrasujeto (medidas repetidas).

\begin{tabular}{lcccc}
\hline \multirow{2}{*}{ Medidas } & Grupo & Media & $\begin{array}{c}\text { Desviación } \\
\text { Estándar }\end{array}$ & N \\
\hline (Tiempo 1) DD_Flopi & DT & 0,240 & 0,523 & 25 \\
\cline { 2 - 5 } & TEL & 0,000 & 0,000 & 25 \\
\hline (Tiempo 3) DD_Bato & DT & 0,400 & 0,645 & 25 \\
\cline { 2 - 5 } & TEL & 0,880 & 1,092 & 25 \\
\hline (Tiempo 5) DD_Lilo & DT & 0,400 & 0,500 & 25 \\
\cline { 2 - 5 } & TEL & 0,880 & 1,013 & 25 \\
\hline
\end{tabular}

Fuente: Elaboración propia.

Como puede verse en las tablas precedentes (Tabla 9, 10 y 11), dentro de la progresión temporal, el grupo DT evidencia mayor producción de DS en el tiempo 1 respecto del grupo TEL, que incluso en la discordancia no muestra producción de este tipo de fallos. En el tiempo 3 (Bato), esta disparidad se mantiene en sustitución, pero se invierte en elisión y discordancia, pues en el cuento de Bato se observa que el promedio de los niños DT es menor que el del grupo TEL. Finalmente, en el tiempo 5 (Lilo), TEL sigue manteniendo una mayor predominancia de desviaciones si se los compara con DT.

En relación con la desviación incorporación (DI), los resultados del análisis descriptivo (Tabla 12) muestran que, dentro de la progresión temporal, el grupo DT no evidencia desviación de incorporación en la primera muestra, a diferencia del grupo TEL (M: 0,040). En el tercer momento, esta disparidad se invierte, pues se observa que el promedio de DT (M: 0,240) es mucho mayor que el grupo TEL (M: 0,080). Sin embargo, en el tiempo 5, vuelve a ser el grupo TEL (M: 0,200) el que evidencia una mayor ocurrencia de DI que el grupo DT (M: 0,080). Esto nos permite plantear que mientras en el grupo DT no es posible observar un patrón de incremento del fallo, el grupo TEL evidencia un aumento en la ocurrencia de desviación asociado con el aumento de la edad. 
Tabla 12.

Descriptivos de la desviación en la subordinación de tipo incorporación (DI) de los grupos DT y TEL en la variable intrasujeto (medidas repetidas).

\begin{tabular}{lcccc}
\hline Medidas & Grupo & Media & $\begin{array}{c}\text { Desviación } \\
\text { Estándar }\end{array}$ & $\mathrm{N}$ \\
\hline (Tiempo 1) DI_Flopi & DT & 0,000 & 0,000 & 25 \\
\cline { 2 - 5 } & TEL & 0,040 & 0,200 & 25 \\
\hline (Tiempo 3) DI_Bato & DT & 0,240 & 0,523 & 25 \\
\cline { 2 - 5 } & TEL & 0,080 & 0,277 & 25 \\
\hline (Tiempo 5) DI_Lilo & DT & 0,080 & 0,277 & 25 \\
\cline { 2 - 5 } & TEL & 0,200 & 0,500 & 25 \\
\hline
\end{tabular}

Fuente: Elaboración propia.

b) Análisis comparativo de los tipos de desviaciones: sustitución, elisión, discordancia e incorporación

El análisis comparativo entre sujetos ha permitido determinar la interacción de ambos grupos en los tipos de desviación en la subordinación durante los tres momentos evaluados.

Tabla 13.

Efectos de interacción por grupo en los tipos de desviación en la subordinación.

\begin{tabular}{lccc}
\hline & $F$ & $p$ & $\eta^{2}{ }_{p}$ \\
\hline Sustitución & $3,362^{\mathrm{a}}$ & $0,049^{\mathrm{a}}$ & 0,065 \\
\hline Elisión & $4,194^{\mathrm{a}}$ & $0,030^{\mathrm{a}}$ & 0,080 \\
\hline Discordancia & $4,039^{\mathrm{a}}$ & $0,026^{\mathrm{a}}$ & 0,078 \\
\hline Incorporación & $2,157^{\mathrm{a}}$ & $0,129^{\mathrm{a}}$ & 0,041 \\
\hline
\end{tabular}

Fuente: Elaboración propia.

En la Tabla 13 se observa que en el caso de las desviaciones de sustitución $(F(2,96)=3,362, p=0,049)$, elisión $(F(2,96)=4,194, p=0,030) \mathrm{y}$ discordancia $(F(2,96)=4,039, p=0,026)$ existe un efecto de interacción estadísticamente significativo, lo que sugiere que la progresión temporal difiere dependiendo del grupo de pertenencia. Por ello, en la Figura 4 se observa que el grupo TEL muestra una trayectoria diferente de la del grupo DT. En cambio, el análisis comparativo entre sujetos en la interacción de ambos grupos en la incorporación arrojó que la estimación del efecto de interacción resultó no ser estadísticamente significa- 
tivo $(F(2,96)=2,157, p=0,129)$. Esto sugiere que la trayectoria temporal en incorporación no difiere dependiendo del grupo de pertenencia.

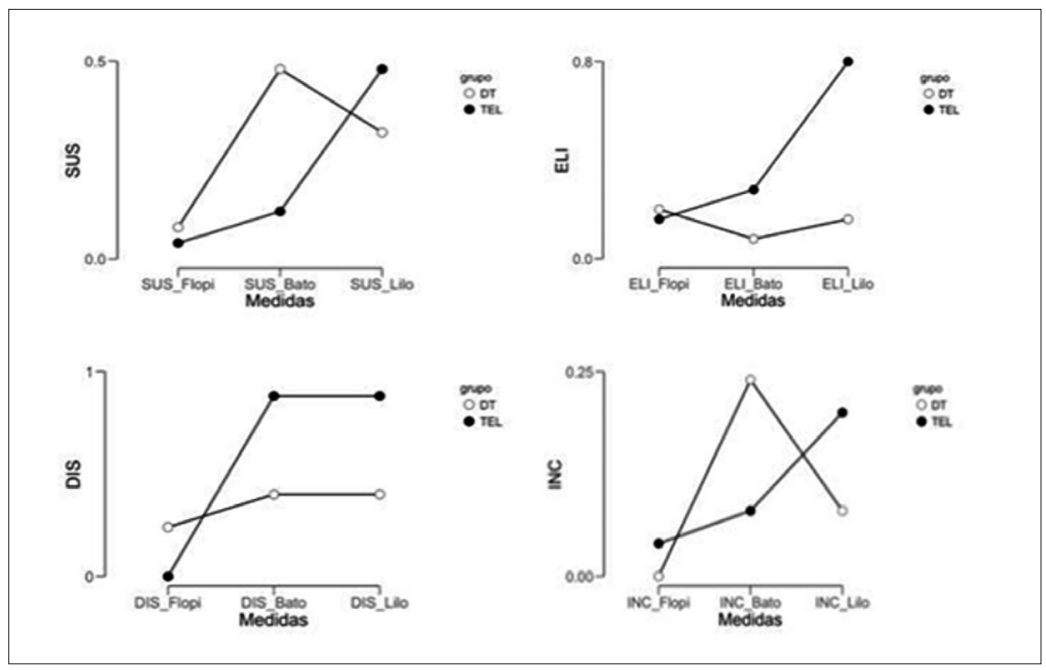

Figura 4. Progresión temporal del desempeño de los grupos DT versus TEL en la desviación de la subordinación tipo sustitución, elisión, discordancia e incorporación.

Nota . SUS = Sustitución; ELI = Elisión; DIS = Discordancia; INC = Incorporación.

\section{Conclusiones}

A partir de los resultados obtenidos en la presente investigación, es posible hacer referencia al cumplimiento de los tres principales objetivos que direccionaron el estudio. En relación con el primer objetivo - determinar si evolutivamente la productividad de la subordinación se mantiene cuantitativamente deficitaria en los niños con TEL en comparación con sus pares con DT- se concluye que en términos evolutivos la baja productividad en la subordinación del grupo con TEL no se mantendría. En la medida en que los niños avanzan en escolaridad, también avanzan en la producción de estructuras subordinadas, desplazando la idea de una producción cuantitativamente menor respecto de la de sus pares con DT, que sí se observa en la temprana infancia. Además, se pudo corroborar que ambos grupos, dentro de la lógica de su productividad, hacen un recorrido parecido. Esto podría sugerir que los sujetos con TEL pueden tener resultados más bajos que los de DT, pero dentro de su propia lógica recorren el mismo camino de la 
adquisición, evidenciando lo planteado por Leonard (2014b) en cuanto a que el niño con TEL no rompe con la adquisición normal, sino que manifiesta solo una mayor dificultad dentro de un perfil similar.

En cuanto al segundo objetivo de este estudio - establecer si existe diferencia en el porcentaje de vínculos interclausulares con fallos o desviaciones en las narraciones producidas por niños con TEL y DT medidos en tres momentos de su escolarización-, los resultados arrojaron que los niños con TEL hacen un recorrido distinto en relación con los niños con DT, evidenciando cada vez más fallos a medida que aumentan en edad. En este sentido, ambos grupos hacen una trayectoria diferente. Es decir, aunque parecen lidiar con los mismos problemas a los cinco años, se observó que el grupo con DT logra superarlos, pues disminuyen de forma gradual a medida que avanzan en su escolarización; en cambio, en el grupo TEL dichas desviaciones aumentan, mostrando una mayor dificultad para lidiar con el desafío que implica la estructura de subordinación. En otras palabras, tanto los niños con TEL como los niños con DT cometen fallos cuando subordinan, no obstante, los primeros producen significativamente más desviaciones que sus pares normotípicos. Estos resultados sugieren que existe una persistencia de la dificultad en la población TEL, a pesar de la escolarización y de la terapia, lo que ya ha sido reportado por otros autores (Durkin, Conti-Ramsden \& Walter, 2011; Simkin \& Conti-Ramsden, 2006; Stothard, Snowling, Bishop, Chipchase \& Kaplan, 1998) lo que parece invalidar la propuesta de que el TEL es una condición transitoria. Se concluye, por tanto, que en términos evolutivos, el grupo TEL sí presenta diferencias en el manejo de ciertos elementos clave cuando subordina en comparación con sus pares con DT, ya que comete significativamente más fallos o desviaciones al vincular dos o más cláusulas subordinadas.

Por último, respecto del tercer objetivo de esta investigación - determinar el tipo de desviación que tiene lugar en la subordinación en las narraciones de los niños con TEL comparado con las de la población con DT - se evidenció que el tipo de fallo en ambos grupos es similar, observándose desviaciones en discordancia (principalmente verbal), elisión (de nexos) y sustitución (de preposiciones). La comparación por tipo de desviación, en la mayoría de los casos nos permite plantear que si bien los niños con DT a los cinco años realizan más desviaciones en 
aspectos interclausulares cuando subordinan que los niños con TEL, la trayectoria les permite superar este fallo en su desarrollo sintáctico, lo que no ocurre con el grupo TEL.

Asimismo, los resultados mostraron que la elisión, la sustitución, la discordancia y la incorporación son elementos cruciales para entender cuáles son los puntos de mayor dificultad que conlleva la vinculación interclausular en el español, pues ambos grupos los cometieron. Este mismo fenómeno también fue observado por Jackson-Maldonado y Maldonado (2017) en un estudio transversal con niños entre los cinco y nueve años de edad. Este hallazgo pareciera corroborar la propuesta de Leonard (2014b) cuando señala que los errores de TEL dejan ver las dificultades mayores que tiene una lengua - en este caso el españoly permiten describir un patrón particular de dificultades gramaticales.

\section{Referencias bibliográficas}

Acosta, V., Moreno, A. M., y Axpe, A. (2014). El estudio de la agramaticalidad en el discurso narrativo del trastorno específico del lenguaje. Revista Onomázein, 29, 119-129. https://doi.org/10.7764/onomazein.2.11

Adani, F., Stegenwallner-Schütz, M., Haendler, Y., \& Zukowski, A. (2016). Elicited production of relative clauses in German: Evidence from typically developing children and children with specific language impairment. First Language, 36(3), 203-227. https://doi.org/10.1177/0142723716648842

Alfaro-Faccio, P., Crespo, N., y Alvarado, C. (2016). Complejidad sintáctica en narraciones de niños con desarrollo típico, trastorno específico del lenguaje y discapacidad intelectual. Sintagma, 28, 27-41. https://doi.org/10.21001/sintagma.2016.28.02

Aguado, G. (1999). Trastorno específico del lenguaje. Retraso del lenguaje y disfasia. Archidona, Málaga: Aljibef.

Aguado, G., Coloma, C. J., Martínez, A. B., Mendoza, E., y Montes, A. (2015). Documento de consenso elaborado por el comité de expertos en TEL sobre el diagnóstico. Revista de Logopedia, Foniatría y Audiología, 35(4), 147-149. https://doi. org/10.1016/j.rlfa.2015.06.004

Alvarado, C., Crespo, N., y Manghi, D. (2016). El impacto de las imágenes en una tarea de recontado: diseño de un cuento ilustrado para niños basado en la gramática visual. Logos: Revis- 
ta de Lingüistica, Filosofía y Literatura, 26(1), 23-39. https:// doi.org/10.15443/RL2602

Auza, A. y Morgan, G. (2013). Uso de preposiciones en el recuento de una historia. Comparación de niños hispanohablantes con y sin trastorno del lenguaje. Infancia y Aprendizaje, 36(1), 3549. https://doi.org/10.1174/021037013804826573

Berman, R. \& Slobin, D. (1994). Relating events in narrative: A crosslinguistic developmental study. Hillsdale: Erlbaum.

Buiza, J. J., Adrián, J. A., González, M., y Rodríguez-Parra, M. J. (2004). Evaluación de marcadores psicolingüísticos en el diagnóstico de niños con trastorno específico. Revista de Logopedia, Foniatría y Audiología, 24(4), 142-155. https://doi. org/10.1016/s0214-4603(04)75797-6

Bustos, A. y Crespo, N. (2014). Comprensión oral de narraciones y producción narrativa: dos medidas a través de una tarea de recontado. Onomázein, 30, 111-126. https://doi.org/10.7764/ onomazein.30.7

Carrasco, A. (1999). El tiempo verbal y la sintaxis oracional. La consecutio temporum. En I. Bosque y V. Demonte (Eds.), Gramática descriptiva de la lengua española (Vol. I) (pp. 3.061-3.128). Madrid: Espasa-Calpe.

Cohen, J. (1988). Statistical power analysis for the behavioral sciences (second edition). Hillsdate: LEA.

Coloma, C. J. (2013). Sintaxis compleja y discurso narrativo en escolares con trastorno específico del lenguaje (TEL). Recuperado de http://0-hera.ugr.es.adrastea.ugr.es/tesisugr/21013044.pdf

Coloma, C. J., Araya, C., Quezada, C., Pavez, M. M., \& Maggiolo, M. (2016). Grammaticality and complexity of sentences in monolingual Spanish speaking children with Specific Language Impairment. Clinical Linguistics \& Phonetics, 30(9), 649-662. http://dx.doi.org/10.3109/02699206.2016.1163420

Coloma, C., Mendoza, E., y Carballo, G. (2017). Desempeño gramatical y narrativo en niños con trastorno específico del lenguaje. Círculo de Lingüística Aplicada a la Comunicación, 69, 6790. http://dx.doi.org/10.5209/CLAC.55314

Colozzo, P., Gillam, R. B., Wood, M., Schnell, R. D., \& Johnston, J. R. (2011). Content and form in the narratives of children with specific language impairment. Journal of speech, language, 
and hearing research, 54(6), 1609-1627. http://dx.doi. org/10.1044/1092-4388(2011/10-0247)

Corpus NIR. (2014). Corpus de narrativas orales de poblaciones DT y $D L D$. Valparaíso: Pontificia Universidad Católica de Valparaíso [no publicado].

Crespo, N., Góngora, B., y Figueroa, A. (2015). La medición por criterio de la narrativa en niños con trastorno específico del lenguaje. Revista de Logopedia, Foniatría y Audiología, 35(3), 99146. http://dx.doi.org/10.1016/j.rlfa.2014.12.003

Crespo, N., Góngora, B., y Manghi, D. (2013). Elementos lingüísticos y cognitivos que se relacionan con el desarrollo de la complejidad sintáctica oral en los primeros años escolares (Informe de avance proyecto Fondecyt 1130420). Valparaíso, Chile.

Crespo, N., Alfaro-Faccio, P., Góngora-Costa, B., Alvarado, C., y Marfull-Villanueva, D. (2020). Perfil sintáctico de niños con y sin trastornos del desarrollo del lenguaje: un análisis descriptivo. Signos, 53 (104), 619-642. DOI: 10.4067/S071809342020000300619

Durkin, K., Conti-Ramsden, G., \& Walker, A. (2011). Txt lang: Texting, textism use and literacy abilities in adolescents with and without specific language impairment. Journal of Computer Assisted Learning, 27(1), 49-57. https://doi.org/10.1111/ j.1365-2729.2010.00397.x

Hincapié, L., Giraldo, M., Lopera, F., Pineda, D., Castro, R., Lopera, J. P., Mendieta, N., ... Lopera, E. (2008). Trastorno específico del desarrollo del lenguaje en una población infantil colombiana. Universitas Psychologica, 7(2), 557-569. Recuperado de https://www.redalyc.org/articulo.oa?id=64770219

Ivanovic, R., Forno, H., Durán, M. C., Hazbún, J., Castro, C., y Ivanovic, D. (2000). Estudio de la capacidad intelectual test de matrices progresiva de Raven en escolares chilenos de 5 a 18 años. Antecedentes generales, normas y recomendaciones. Revista de Psicología General y Aplicada, 53(1), 5-30. Recuperado de https://dialnet.unirioja.es/servlet/articulo?codigo $=2356766$

Jackson-Maldonado, D. y Maldonado, R. (2015). La complejidad sintáctica en niños con y sin Trastorno Primario de Lenguaje. En I. Rodríguez Sánchez y E. Vázquez (Eds.), Estudios de lingüística funcional (pp. 235-301). Santiago de Querétaro: Universidad Autónoma de Querétaro. 
Jackson-Maldonado, D. \& Maldonado, R. (2017). Grammaticality differences between Spanish-speaking children with specific language impairment and their typically developing peers. International Journal of Language \& Communications Disorders, 52(6), 750-765. https://doi.org/10.1111/14606984.12312

Leonard, L. B. (2014a). Children with specific language impairment (second edition). London: MIT Press.

Leonard, L. B. (2014b). Specific language impairment across languages. Child Development Perspectives, 8(1), 1-5. https://doi. org/10.1111/cdep.12053

Mendoza, E. (2001). Trastorno específico del lenguaje (TEL). Madrid: Psicología Pirámide.

Mota, S. (2015). ¿Por qué se usa "recursión" cuando se quiere significar auto-inclusión?: clarificaciones conceptuales sobre la recursión en el programa chomskiano. RLA. Revista de Lingüística Teórica y Aplicada, 53(2), 171-191. https:// dx.doi.org/10.4067/s0718-48832015000200008

Nippold, M. (2004). Research on later language development. International perspectives. En R. Berman (Ed.), Language development across childhood and adolescence (pp. 1-8). Philadelphia: John Benjamins.

Owens, R. (1995). Language disorders: A functional approach to assessment and intervention (second edition). Boston: Allyn and Bacon.

Pavez, M. M. (2010). Test exploratorio de gramática española de A. Toronto. Aplicación en Chile (tercera edición). Santiago de Chile: Ediciones Pontificia Universidad Católica de Chile.

Pavez, M. M., Coloma, C. J., y Maggiolo, M. (2008). El desarrollo narrativo en niños. Una propuesta práctica para la evaluación y la intervención en niños con trastornos de lenguaje. Barcelona: Lexus.

Pavez, M. M., Maggiolo, M., y Coloma, C. J. (2009). Test para evaluar procesos de simplificación fonológica. TEPROSIF-R. (tercera edición). Santiago de Chile: Ediciones Pontificia Universidad Católica de Chile.

Perea, F. J. y Martínez-Atienza, M. (2013). En torno a la combinatoria de tiempos verbales en español. Language Design 15, 29-59. 
Recuperado de http://elies.rediris.es/Language_Design/ LD15/LD15_02_Perea_y_Martinez.pdf

Peterson, C. \& McCabe, A. (1983). Developmental psycholinguistics: Three ways of looking at a child's narrative. New York: Plenum.

Raven, J. C. (2005). Test de matrices progresivas. Escala coloreada. Cuaderno de Matrices/Series A, AB y B. Buenos Aires: Paidós.

Real Academia Española/Asociación de Academias de la Lengua Española, RAE/ASALE. (2009). Nueva gramática de la lengua española. Morfología y sintaxis. Madrid: Espasa.

Rojo, G. y Veiga, A. (1999). El tiempo verbal. Los tiempos simples. En I. Bosque y V. Demonte (Directores.), Gramática descriptiva de la lengua española (vol. 2), (pp. 2867-2934). Madrid: Espasa Calpe/Real Academia Española, Colección Nebrija y Bello.

Simkin Z. \& Conti-Ramsden G. (2006). Evidence of reading difficulties in subgroups of children with specific language impairment. Child Language Teaching and Therapy, 22(3), 315-331. https://doi.org/10.1191/0265659006ct310xx

Stein, N. \& Glenn, C. (1979). An analysis of story comprehension in elementary school children. En R. O. Freedle (Ed.), Advances in discourse processes: New directions in discourse processing (vol. 2) (pp. 53-120). Norwood: Ablex.

Stothard, S., Snowling, M., Bishop, D., Chipchase, B., \& Kaplan, C. (1998) Language impaired preschoolers: A follow-up into adolescence. Journal of Speech and Hearing Research, 41(2), 407-418. https://doi.org/10.1044/jslhr.4102.407

Van der Lely, H. K. J. (1998). SLI in children: Movement, economy, and deficits in the computational-syntactic system. Language Acquisition, 7(2-4), 161-192. https://doi.org/10.1207/ s15327817la0702-4_4

Van der Lely, H. K. J. (2005). Grammatical-SLI and the computational grammatical complexity hypothesis. Frequences, 17(3), 1320. Recuperado de https://scholar.harvard.edu/vanderlely/ publications/grammatical-sli-and-computational-grammatical-complexity-hypothesis

Veiga, A. y Mosteiro, M. (2006). El modo verbal en cláusulas condicionales, causales, consecutivas, concesivas, finales y adverbiales de lugar, tiempo y modo. Salamanca: Ediciones Universidad de Salamanca. 
Villanueva, P., De Barbieri, Z., Palomino, H. M., y Palomino, H. (2008). Alta prevalencia de trastorno específico de lenguaje en isla Robinson Crusoe y probable efecto fundador. Revista Médica de Chile, 136(2), 186-192. http://dx.doi.org/10.4067/S003498872008000200007 